\title{
Slide ID Examined by Pathologist
}

National Cancer Institute

\section{Source}

National Cancer Institute. Slide ID Examined by Pathologist. NCI Thesaurus. Code

C162690.

The identifier for an slide that has been examined by a pathologist. 